\title{
Changes in shape and color of an embodied virtual arm modulate pain experience in healthy subjects.
}

Marta Matamala-Gomez ${ }^{1,2}$, Birgit Nierula ${ }^{1,2}$, Mel Slater ${ }^{2,3}$, Maria V. Sanchez-Vives ${ }^{1,2,3}$

${ }^{1}$ IDIBAPS, Barcelona, Spain. ${ }^{2}$ Event Lab, University of Barcelona, Spain. ${ }^{3}$ ICREA, Barcelona, Spain.

\section{Background}

A virtual body can be "embodied" and be felt as own when congruent multisensory correlations are provided. The visual aspect of such an "owned" virtual arm has been shown to modify pain threshold in healthy subjects. However, it is not well known how this can be used to reduce pain in chronic pain patients with a distorted body image.

\section{Aim}

In this study, we investigated how shape and colour of the virtual body impact the pain experience in healthy subjects when a threat approaches and eventually contacts the virtual body.

\section{Participants}

We conducted a $3 \times 2$ within-subjects study with 30 right-handed healthy subjects. Three subject had been excluded from further analysis due to extreme high z-score in the SCR data. Finally, 27 right-handed subjects participated in this study (Mean Age $\pm S D=$ 24.70 \pm 1.11 ; Mean Edimburgh scale $\pm S D=$ $68.68 \pm 1.1)$.

\section{Procedures}

In this study, there were two main factors while participants were embodied in a virtual body. The first factor was 'Virtual arm', with three different representations of the virtual arm: virtual arm normal position (control) virtual arm distorted position (distorted) and virtual arm distorted position and redcoloured (reddened-distorted) (Figure 1).
B)

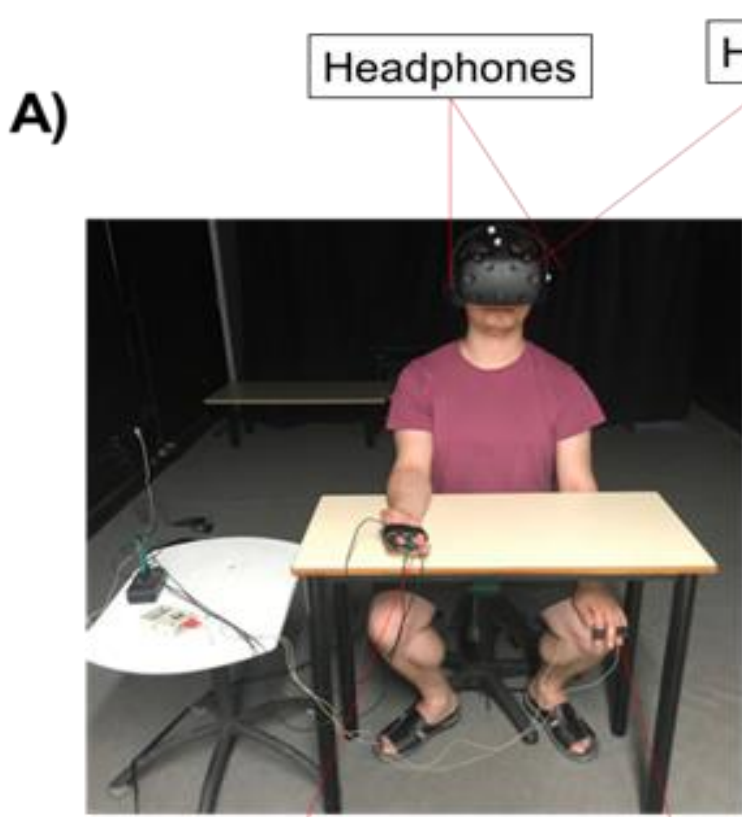

Visuo-tactile stimulation



C)

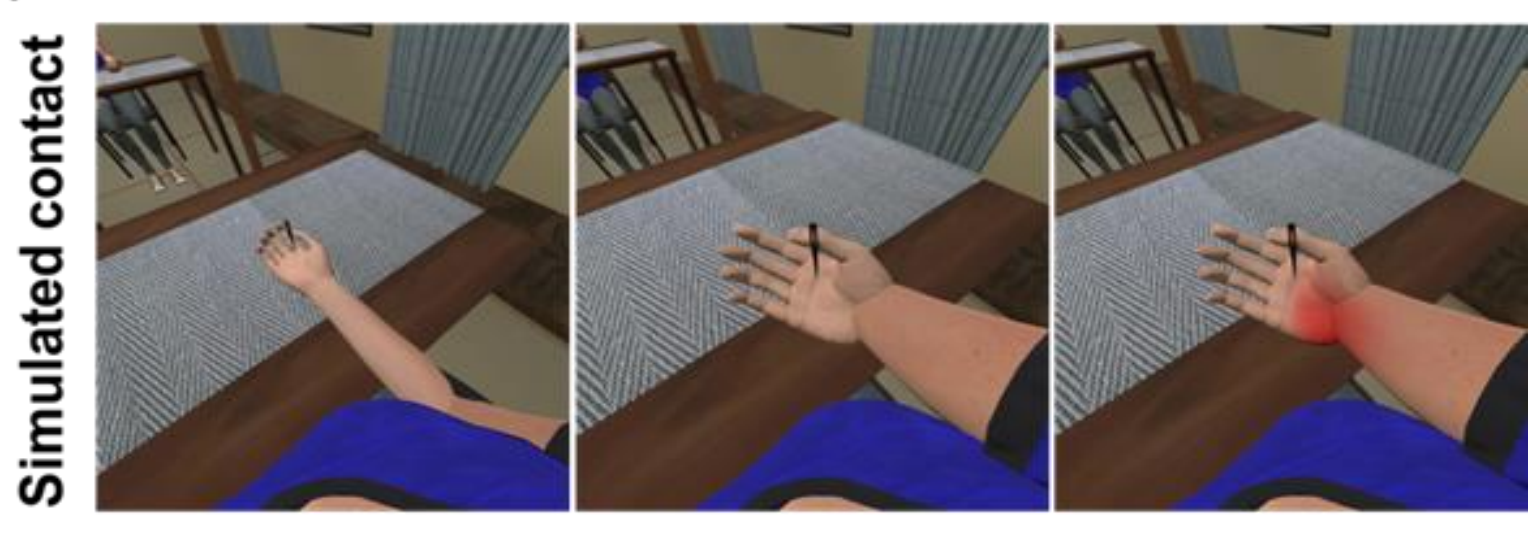

Figure 1. A) Participants position during the experimental session. B) Three different representation of the virtual arm during the real contact condition. C) Three different representation of the virtual arm during the simulated contact condition.

In all cases, the virtual arm was co-located with the real arm. The second factor was 'Threatening stimulus contact', with two different types of contact of the threatening stimulus: the stimulus that contact the skin (real contact) and stimulus that approach but not touch the skin (simulated contact). In each condition, the Skin Conductance Response (SCR), the level of pain/discomfor and ownership were assessed after each threat to the virtual arm

Each condition was repeated 3 times. Hence, participants completed a total of 18 virtual arm and threating stimulus exposures.

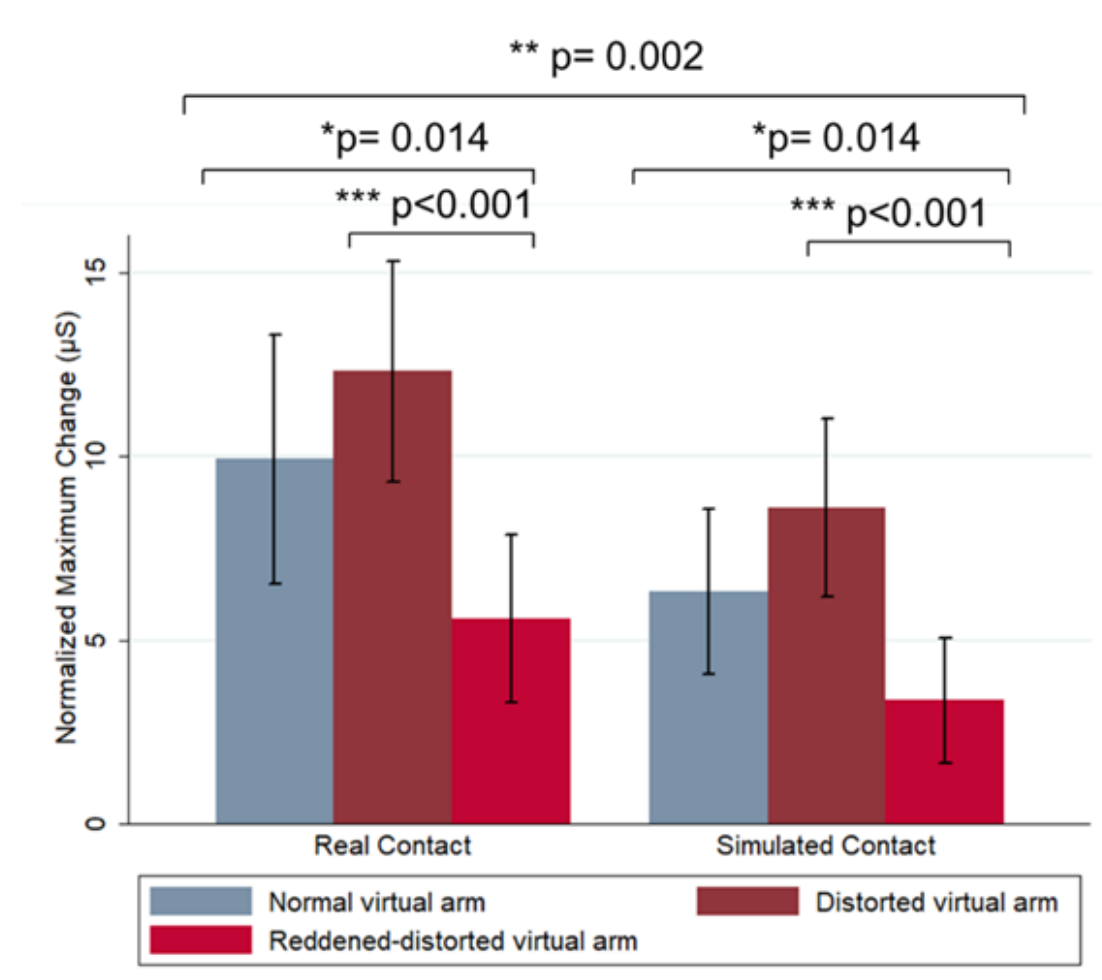

Figure 2. SCR in the three different representation of the virtual arm after each threat stimulus condition.
Results

Our findings demonstrate:

- The distorted virtual arm enhances the SCR to the threats with respect to those of the control arm. Interestingly, the reddened-distorted virtual arm did not show a significant increase of the SCR to the threat. We discuss the possibility that the redness of the arm induces a furthe anticipation of the pain sensation (Figure2).

- There was a positive relationship between the level of ownership over the distorted and reddened-distorted virtual arms with the level of pain/discomfort but not in the control arm (Figure 3).

- Further, real contact of the threat significantly enhances SCR and pain/discomfort ratings (Figure2 and 3).

\section{Conclusion}

Pain experience relay on the multisensory representation of the body and this principle could be exploited for reducing pain experience in chronic pain patients.
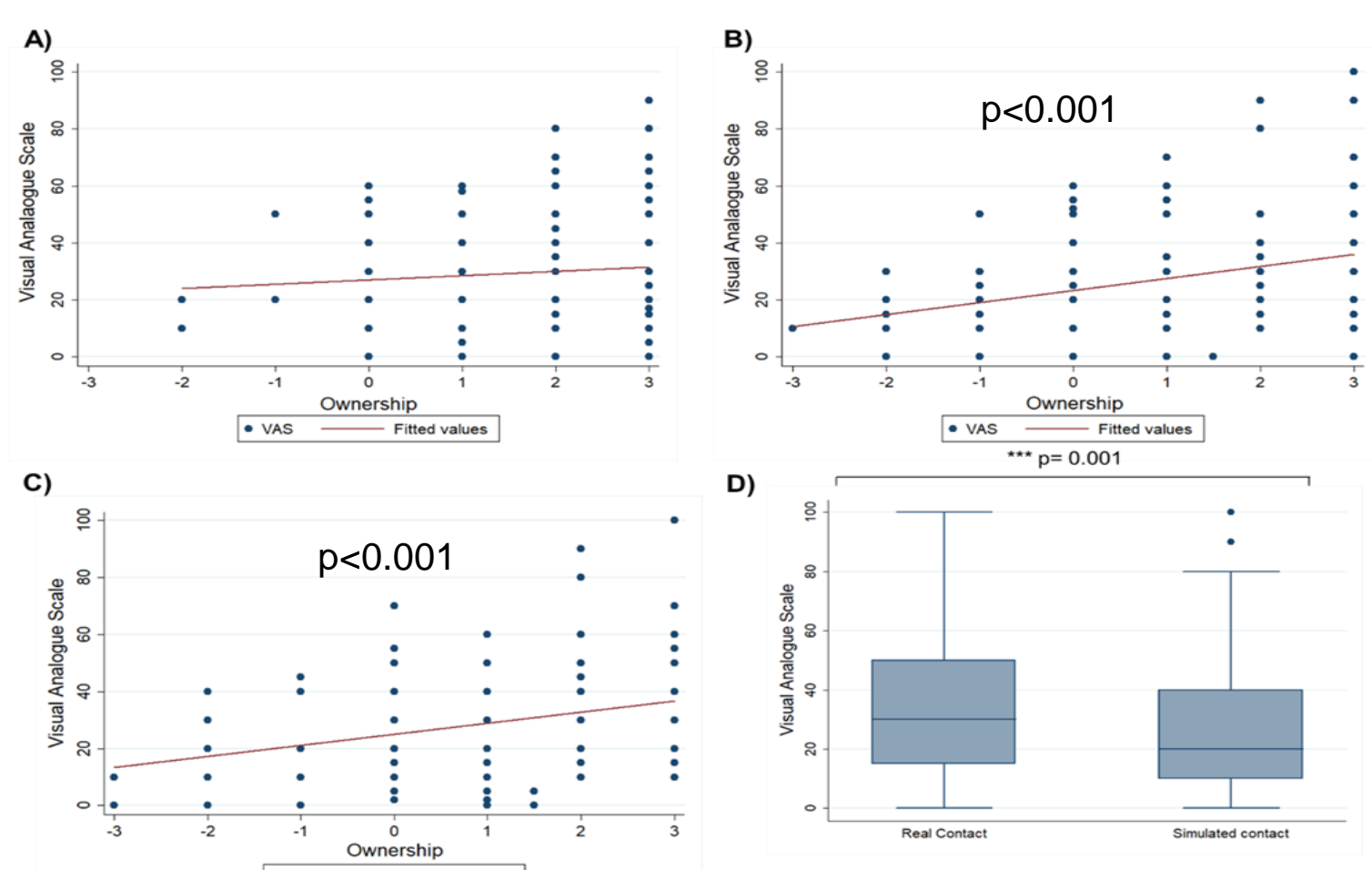

Figure 3. $A, B, C)$ Relationship between ownership over the different virtual arm representation and pain/disconfort. D) Pain/disconfort ratings after each threat stimulus condition. 